\title{
SÍNDROME DE SHEEHAN: DESCRIPCIÓN DE UN CASO CLÍNICO Y REVISIÓN DE LA LITERATURA
}

\section{Sheehan's syndrome: a case report and literature review}

\author{
Eduardo Contreras-Zúñiga, M.D. *, Ximena Mosquera-Tapia, M.D.**, \\ María Cristina Domínguez-Villegas, M.D.***, Erika Parra-Zúñiga, M.D.***** \\ Recibido: junio 4/09 - Aceptado: octubre 26/09
}

\section{RESUMEN}

Introducción: la necrosis hipofisaria posparto o síndrome de Sheehan se presenta secundaria a una hemorragia obstétrica, ocasionando un colapso circulatorio intenso el cual, a su vez, predispone a la isquemia de la hipófisis aumentada de tamaño.

Objetivo: presentar un caso clínico de una paciente quien consultó por manifestaciones tardías de este síndrome y hacer una revisión de la literatura.

Metodología: se buscó información en la bases de datos de PubMed/MEDLINE, Cochrane y SciELO; así como en referencias de artículos de revista y textos principalmente de los últimos cinco años. Seguidamente, se tomaron los siete artículos más relevantes según la calificación de la revista en donde fueron publicados.

Resultados: la característica clínica del síndrome de Sheehan que se manifiesta con mayor frecuencia es la incapacidad para lactar debido a una producción insuficiente de prolactina. Otras manifestaciones tardías incluyen: secreción inadecuada de la hormona antidiurética y deficiencias en la secreción de cortisol, de hormona

\footnotetext{
* Medicina Interna. Fellowship en Cardiología, Universidad del Valle. Cali (Colombia). Correspondencia: Calle 4 No. 65-14. Correo electrónico: edo11@hotmail.com

** Ginecología y Obstetricia, Universidad del Valle. Cali (Colombia).

*** Médico y Cirujano, Universidad Libre, Fundación Salamandra. Cali (Colombia).

**** Médico y Cirujano, Universidad Libre, Coomeva E.P.S. Cali (Colombia)
}

tiroidea y de gonadotrofinas. Es importante que el obstetra y el médico general sepan reconocer este síndrome, ya que la pérdida aguda del funcionamiento de la adenohipófisis puede ser de mal pronóstico si no se realiza un reemplazo hormonal adecuado. Además, el buen control hormonal evita o disminuye las complicaciones metabólicas y cardiovasculares.

Conclusión: el síndrome de Sheehan es una enfermedad poco frecuente, la cual es difícil de diagnosticar tempranamente. Asimismo, requiere una terapia de reemplazo hormonal con controles permanentes clínicos y paraclínicos.

Palabras clave: panhipopituitarismo, síndrome de Sheehan, necrosis hipofisiaria.

\section{SUMMARY}

Introduction: postpartum pituitary necrosis, or Sheehan's syndrome, occurs following obstetric haemorrhage which causes intense circulatory collapse, thereby predisposing pituitary ischemia leading to this organ becoming enlarged (the posterior pituitary is generally affected). This document presents a clinical case of a patient who consulted due to late manifestations of this syndrome. A literature review is also made.

Objective: presenting the case of a patient who consulted for late manifestations of Sheehan's syndrome and reviewing the pertinent literature. 
Methodology: information was sought in the PubMed / Medline, Cochrane, SciELO databases and references from articles in journals and texts (mainly from the last five years) were also taken into account. The seven most relevant articles were taken according to the impact of the journal in which they were published.

Results: the most frequently found clinical characteristic was an inability to lactate due to insufficient prolactin production. The most common clinical feature found was an inability to breastfeed due to insufficient prolactin production. Other manifestations included inappropriate antidiuretic hormone secretion and cortisol, thyroid hormone and gonadotropin secretion deficiencies. Obstetricians and GPs should be able to recognise this syndrome and know about its management as the acute loss of adenohypophysis functioning may have a poor prognosis without timely and suitable hormone replacement. Good hormone control prevents or decreases metabolic and cardiovascular complications.

Conclusion: this is a rare syndrome in which early diagnosis is difficult and requires hormone replacement therapy with ongoing clinical and laboratory controls.

Keywords: panhypopituitarism, Sheehan's syndrome, pituitary necrosis.

\section{INTRODUCCIÓN}

El síndrome de Sheehan, descrito por primera vez en 1937 por HL Sheehan, ${ }^{1}$ es el nombre que se le dio a la necrosis de la glándula hipófisis como consecuencia de una hemorragia obstétrica acompañada de un colapso circulatorio intenso que conlleva a la aparición de hipopituitarismo en el posparto. Las áreas necróticas de la adenohipófisis posteriormente se organizan y forman una cicatriz fibrosa. La destrucción extensa de estas células resulta en un grado variable de hipopituitarismo en un tiempo muy variable que oscila de días a años. Por consiguiente, es importante para el obstetra y el médico general reconocer este síndrome, ya que la pérdida aguda del funcionamiento de la adenohipófisis en un período de tiempo variable después del parto, puede ser fatal si no se realiza un reemplazo hormonal y se continúa posteriormente, y por el resto de la vida, con tratamiento hormonal indicado según la deficiencia que se haya generado. Por otra parte, el hipopituitarismo tratado de manera inadecuada produce una aterosclerosis prematura, aumentando la mortalidad en este tipo de pacientes. ${ }^{2,3}$ De esta manera, el objetivo de la presentación de este caso clínico es hacer una revisión de la literatura respecto a la frecuencia, fisiopatología, diagnóstico y tratamiento de la enfermedad.

\section{DESCRIPCIÓN DEL CASO CLÍNICO}

Mujer de 32 años quien consultó por cuadro clínico de 3 semanas de evolución consistente en enlentecimiento, palidez, debilidad, mareo, alteraciones en la agudeza visual, cefalea y diaforesis, y posterior empeoramiento de los síntomas en las últimas 48 horas por lo cual decidió asistir a consulta. Al ingreso se observó una paciente en regulares condiciones: hidratada; TA 85/40 mmHg; Frecuencia Cardíaca (FC) 64 latidos por minuto; Frecuencia Respiratoria (FR) 18 respiraciones por minuto; no ingurgitación yugular; campos pulmonares ventilados sin ruidos sobre agregados; ruidos cardíacos rítmicos sin soplo; abdomen blando, depresible y no doloroso a la palpación; sin presencia de edema; piel seca y áspera; con ausencia de vello axilar y marcada disminución del vello púbico; alerta bradipsíquica y sin déficit motor o sensitivo. Ante esto, se inició manejo con líquidos endovenosos, obteniendo mejoría sintomática y en tensión arterial. Además, se interrogó a la paciente quien informó de caída de cabello, incremento de peso y amenorrea. Entre los antecedentes se encontró un parto 15 meses atrás con aparente sangrado importante, por lo cual se comenzó a sospechar una necrosis hipofisaria posparto. 
Se solicitaron exámenes de laboratorio que fueron informados de la siguiente manera: glicemia $78 \mathrm{mg} / \mathrm{dL}$, leucocitos $8.700 \times$ mm$^{3}$, N 72\%, L 14\%, Hb $8,23 \mathrm{~g} / \mathrm{dL}$, Hto $22,6 \%$, plaquetas $152.000 \mathrm{x} \mathrm{mm}^{3}, \mathrm{Na}$ $128 \mathrm{mmol} / \mathrm{L}$ (normal 135-145 mmol/L), K4,5 mmol/L (normal 3,5-5,0 mmol/L), Cl $116 \mathrm{mmol} / \mathrm{L}$ (normal 95-120 mmol/L), creatinina 1,36 mg/Dl, BUN $12 \mathrm{mg} / \mathrm{dL}, \mathrm{TSH}$ tercera generación 5,1 uUI/ml (normal 0,4 a 5,0 uUI/mL), T4 libre 0,27 ng/dL (normal 0,80 a $1,90 \mathrm{ng} / \mathrm{dL}), \mathrm{T} 3$ total $<0,40 \mathrm{ng} / \mathrm{mL}(0,84$ a $1,72 \mathrm{ng} / \mathrm{dL})$, cortisol basal 1,1 ug/dL (normal 5,0 a 25 ug/dL), FSH 4,3 mUI/mL (normal 21,7 a $153 \mathrm{mUI} / \mathrm{mL}$ ), LH 1,35 mUI/L (normal 11,3 a 39,8 mUI/L), prolactina $<0,50 \mathrm{ng} / \mathrm{mL}$ (normal 1,90 a $25 \mathrm{ng} / \mathrm{mL}$ ).

Inmediatamente después, se inició manejo con restricción de líquidos, hidrocortisona 100 mg IV cada 8 horas por 3 días, para continuar con prednisolona oral, levotiroxina $100 \mu \mathrm{g} / \mathrm{día}$, fluorocortisona $0,1 \mathrm{mg} /$ día, carbonato de calcio 600 $\mathrm{mg}$ /día, calcitriol 0,5 mg/día, omeprazol $20 \mathrm{mg}$ / día, estrógenos y progestágenos. Adicionalmente, se tomó una Resonancia Magnética Nuclear (RMN) del cerebro (figura 1), observándose "silla turca vacía”.

Más adelante, la paciente presentó mejoría clínica y fue dada de alta para continuar controles de manera ambulatoria. Dos meses después fue evaluada, observándose una mejoría clínica importante. En general, los exámenes paraclínicos mostraron una TSH y un cortisol en rangos normales; por lo cual se le disminuyó la dosis de prednisolona para continuar con 7,5 mg de base.

Figura 1. Glándula colapsada sobre el piso de la silla turca.

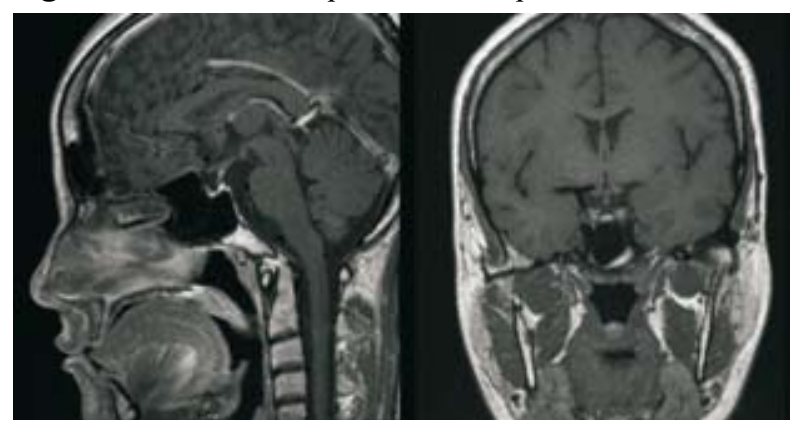

\section{METODOLOGÍA}

Se buscó información en la bases de datos PubMed/ MEDLINE, Cochrane y SciELO, con base en los términos panhipopituitarismo, síndrome de Sheehan y necrosis hipofisiaria; así como en referencias de artículos de revistas y textos principalmente de los últimos cinco años. Seguidamente, se tomaron los ocho artículos más relevantes según la calificación de la revista en donde cada uno fue publicado.

\section{DISCUSIÓN}

La frecuencia del síndrome de Sheehan ha disminuido en los últimos años, principalmente en los países desarrollados, debido a un mejor cuidado obstétrico. Actualmente, el síndrome es considerado una consecuencia poco común de un cuadro hemorrágico obstétrico. Sin embargo, es un cuadro a tener en cuenta en pacientes que presentan hemorragia posparto ya que se ha descrito que 25\% a 32\% de las pacientes con sangrado severo asociado con el parto presentará algún grado de panhipopituitarismo., ${ }^{2,4}$

En referencia a la patogénesis de este síndrome, no hay duda de que el proceso básico es el infarto secundario a la ausencia de flujo sanguíneo en el lóbulo anterior de la glándula pituitaria. No obstante, no se tiene la certeza de si éste es resultado de vasoespasmo, trombosis o compresión vascular. Al final del embarazo, la glándula pituitaria está significativamente aumentada de tamaño (principalmente por hiperplasia de las células secretoras de prolactina); ${ }^{3}$ por lo que este aumento de tamaño podría ejercer un efecto de compresión de los vasos sanguíneos, lo que los haría más susceptibles a la hipoperfusión con bajo aporte de oxígeno y otros nutrientes a la glándula en situación de colapso circulatorio. De otra parte, se ha planteado que las células de la adenohipófisis en la mujer embarazada pueden ser más susceptibles de lo normal a la isquemia, en lugar de ser el resultado de ambos factores. ${ }^{3}$ Por su parte, otros autores han sugerido que la necrosis pudiera ser secundaria a trombosis primaria debido a la agregación plaquetaria en las células endoteliales lesionadas. ${ }^{3}$ Por el contrario, la 
presencia o ausencia de vasoespasmo no ha podido ser confirmada por investigación microscópica. ${ }^{5}$

Por otro lado, la glándula pituitaria no puede regenerarse, por lo cual no es posible que se formen células nuevas para reemplazar las células necróticas que son substituidas por células cicatrizales. ${ }^{6}$ La función glandular puede ser mantenida por aproximadamente $50 \%$ de la glándula, pero un hipopituitarismo parcial o total se presenta cuando hay pérdida de 75\% y 90\% de las células de la adenohipófisis, respectivamente. ${ }^{5}$ Las pacientes tienen diferentes grados de hipopituitarismo desde el panhipopituitarismo hasta deficiencias pituitarias selectivas. ${ }^{6}$

Además, el diagnóstico del síndrome de Sheehan puede ser difícil. Éste se centra en la evidencia clínica de hipopituitarismo en una mujer con historia de hemorragia posparto. La falla en la lactancia o las dificultades para ésta son los síntomas iniciales comunes de este síndrome. Asimismo, muchas mujeres también reportan amenorrea u oligomenorrea después del parto. ${ }^{6}$ En algunos casos, el diagnóstico no se realiza sino hasta años después, cuando ocurren alteraciones secundarias al hipopituitarismo como el hipotiroidismo secundario o la insuficiencia adrenal secundaria; éste último, consecuencia de un situación de estrés como el producido por infecciones o cirugías. ${ }^{6,7}$

La deficiencia en hormonas específicas de la anterohipófisis causa una variedad de síntomas. Por ejemplo, la deficiencia de corticotropina produce debilidad, fatiga, hipoglicemia o letargo; la insuficiencia de gonadotropina usualmente provoca la aparición de amenorrea, oligomenorrea, oleadas de calor o disminución en la libido; y la carencia de la hormona del crecimiento produce síntomas vagos incluyendo fatiga, disminución en la masa muscular y deterioro en la calidad de vida. ${ }^{5,6}$ Adicionalmente, se encontrarán niveles bajos de hormonas pituitarias (hormona luteinizante, corticotropina y tirotropina) al igual que hormonas en las que ésta actúa (cortisol y tiroxina). Es por esto que las pruebas de estimulación (hipoglicemia inducida por insulina o metyrapone) son a veces necesarios para el diagnóstico en la fase aguda o en situaciones en las que se sospecha una deficiencia parcial. ${ }^{6}$

Por otro lado, la hiponatremia es poco común en la presentación aguda del síndrome. ${ }^{7}$ Aún así, hay varios mecanismos por los cuales el hipopituitarismo puede resultar en hiponatremia. El hipotiroidismo puede causar disminución en el aclaramiento del agua libre con la hiponatremia subsecuente. La deficiencia de glucocorticoides puede también causar disminución en el aclaramiento del agua libre independiente de vasopresina. El hipopituitarismo por sí mismo puede estimular la secreción de vasopresina y causar una severa secreción inapropiada de la hormona antidiurética, la cual puede también producir hiponatremia. Ahora bien, el nivel de potasio en estas situaciones es normal, debido a que la producción adrenal de aldosterona no es dependiente de la pituitaria. ${ }^{6,7}$

De otra parte, la tomografía computarizada o una resonancia magnética usualmente no son útiles en la fase aguda. La imagen característica es la de "silla turca vacía”, la cual aparece varios meses posteriores al evento hemorrágico., ${ }^{4,7}$

El diagnóstico diferencial se hace con causas de hipofisitis linfocitaria autoinmune que han sido descritas, ocasionalmente asociadas con el embarazo. ${ }^{6}$ Si no se realiza tratamiento, este desorden puede llevar al hipopituitarismo derivado de la destrucción masiva de la glándula pituitaria y su reemplazo por tejido fibrótico. Una vez esta organización ha tenido lugar, los signos histológicos pueden ser similares a los del síndrome de Sheehan, aunque la patogénesis es totalmente diferente. ${ }^{5,6}$

Por otra parte, el hipotiroidismo secundario es clínicamente idéntico al hipotiroidismo primario. No obstante, los pacientes con hipotiroidismo causado por hipopituitarismo tienen niveles bajos de T3 y T4 con valores normales o inapropiadamente bajos de TSH. ${ }^{6}$

Ahora bien, el tratamiento requerido para el manejo del síndrome incluye el reemplazo hormonal inicialmente con hidrocortisona, el cual se debe a que el tratamiento con tiroxina puede exacerbar 
una deficiencia de glucocorticoides y, teóricamente, inducir una crisis adrenal. Posteriormente, se realiza el reemplazo con hormona tiroidea y estrógenos con o sin progestágenos dependiendo si la paciente tiene o no útero. La dosis estándar de hidrocortisona es 20 mg al día (15 mg en la mañana y 5 mg en la tarde). ${ }^{6}$ Adicionalmente, se realiza reemplazo con las demás hormonas dependiendo de la deficiencia. La dosis se ajustará según los resultados de los laboratorios.

\section{CONCLUSIÓN}

El síndrome de Sheehan es una complicación poco frecuente en las mujeres que presentan hemorragia posparto, pero debido a la magnitud de alteraciones hormonales que éste puede generar, debe tenerse presente como parte de la sospecha diagnóstica en caso de tener pacientes con deficiencia hormonal sintomática, mejorando así la calidad de vida y disminuyendo el riesgo de muerte oportunamente.

\section{REFERENCIAS}

1. Sheehan HL. Postpartum necrosis of anterior pituitary. J Pathol Bact 1937;45:189-214.

2. Soares DV, Conceição FL, Vaisman M. Aspectos diagnósticos e terapêuticos da síndrome de Sheehan. Arq Bras Endocrinol Metab 2008;58:872-8.

3. Yamada M, Mori M. Mechanisms related to the pathophysiology and management of central hypothyroidism. Nat Clin Pract Endocrinol Metab 2008;4:683-94.

4. Feinberg EC, Molitch ME, Endres LK, Peaceman AM. The incidence of Sheehan's syndrome after obstetric hemorrhage. Fertil Steril 2005;84:975-9.

5. Ozkan Y, Colak R. Sheehan syndrome: clinical and laboratory evaluation of 20 cases. Neuro Endocrinol Lett 2005;26:257-60.

6. Kovacks K. Sheehan syndrome. Lancet 2003;361:520-2.

7. Shrager S, Sabo L. Sheehan syndrome: a rare complication of postpartum hemorrhage. J Am Board Fam Pract 2001;14:389-91. 